\title{
Thermomagnetic properties and Debye screening for magnetized quark-gluon plasma using the extended self-consistent quasiparticle model
}

\author{
Sebastian Koothottil $\odot^{*}$ and Vishnu M. Bannur \\ Department of Physics, University of Calicut, Kerala 673635, India
}

(Received 7 January 2020; revised 17 June 2020; accepted 13 July 2020; published 23 July 2020)

\begin{abstract}
The thermomagnetic behavior of quark-gluon plasma has recently received a great deal of attention. In this work we make use of the extended self-consistent quasiparticle model to study the thermodynamic properties, magnetic response, and screening properties of magnetized (2+1)-flavor quark-gluon plasma (QGP). The system is considered as a noninteracting system of quasiparticles with masses depending on both temperature and magnetic fields. This allows us to obtain the equation of state of the system and other thermodynamic properties such as the speed of sound. We use the extended self-consistent model to obtain the magnetization and show that QGP has a paramagnetic nature. In addition, we study the pressure anisotropy and calculate the transverse pressure. The obtained anisotropic pressure may be used in hydrodynamic studies of magnetized QGP produced in heavy-ion collisions. We then study the total pressure in both directions, taking pure-field contributions into consideration. Finally we examine the screening properties of magnetized QGP in the longitudinal direction by calculating the Debye screening mass. We go on to compare our results for Debye mass with the results from other approaches.
\end{abstract}

DOI: 10.1103/PhysRevC.102.015206

\section{INTRODUCTION}

Quark-gluon plasma (QGP), the state of matter believed to have existed shortly after the big bang, has been successfully created in high-energy collisions [1]. The charged ions can produce large magnetic fields reaching up to $e B \approx(1-15) m_{\pi}^{2}$ $[2,3]$ during off-central collisions. Magnetic fields created in this manner may exist only for a short while but can be stationary during this time [4-7]. The theoretical tools used to study QGP need modifications to incorporate effects of external magnetic fields and there has been flurry of research activity in this area [8-33]. Measurements at the Large Hadron Collider [34], along with those at relativistic heavy ion collider energies [35], are capable of providing new insights that can constrain the theoretical modeling. The equation of state has a significant impact on the evolution of QGP [36]. The study of the equation of state of magnetized QGP is relevant in the contexts of cosmology [37] and strongly magnetized neutron stars too [38-42]. The investigation of the behavior of magnetized QGP is, therefore, of importance [43]. There are different approaches to studying the effect of magnetic fields on QGP [8,16,44-46].

*sebastian_dop@uoc.ac.in

Published by the American Physical Society under the terms of the Creative Commons Attribution 4.0 International license. Further distribution of this work must maintain attribution to the author $(s)$ and the published article's title, journal citation, and DOI. Funded by $S C O A P^{3}$.
In Ref. [45], we developed the extended self-consistent quasiparticle model and studied the behavior of energy density, longitudinal pressure, and entropy density of magnetized 2-flavor QGP. In this work, we apply the extended quasiparticle model to magnetized (2+1)-flavor QGP. We use it to study the behavior of longitudinal pressure, speed of sound, magnetization, pressure anisotropy, and Debye screening. We start with the study of thermodynamics by obtaining the equation of state and calculating the speed of sound in the medium. We then make use of our model to study the magnetic response of QGP by finding the magnetization and show that the system exhibits paramagnetic behavior. Using the calculated magnetization, we go on to study the anisotropy between longitudinal and transverse pressures caused by the magnetization acquired by the system along the field direction. We bring out the dependence of transverse pressure on temperature and magnetic fields. Then we study the behavior of the total longitudinal and transverse pressure, including the pure-field or Maxwell contributions. Finally, we examine the screening properties of magnetized QGP in the longitudinal direction by calculating the Debye screening mass. As a consistency check, we compare the Debye mass obtained within our formalism with the corresponding result using perturbation techniques and examine how well they agree, especially at high temperatures.

The paper is organized as follows. In Sec. II, we summarize the extended self-consistent quasiparticle model developed in Ref. [45]. In Sec. III, we apply this formulation for the case of $(2+1)$-flavor QGP and obtain its equation of state and speed of sound in the medium. Section IV involves the calculation of the magnetization of QGP. In Sec. V we study the pressure anisotropy. Section VI deals with the pure-field or Maxwell 
contribution to the total pressure. In Sec. VII we calculate the Debye screening mass of magnetized QGP and compare our results with other results. In Sec. VIII, we discuss the results obtained and conclude in Sec. IX.

\section{THE EXTENDED SELF-CONSISTENT QUASIPARTICLE MODEL}

In the extended self-consistent quasiparticle model, the thermal properties of interacting real particles are modeled by noninteracting quasiparticles with thermomagnetic masses [45].

In the self-consistent quasiparticle model [47-52], the thermal mass is defined to be proportional to the plasma frequencies as

$$
m_{g}^{2}=\frac{3}{2} \omega_{p}^{2} \text { and } m_{q}^{2}=\left(m_{0}+m_{f}\right)^{2}+m_{f}^{2} .
$$

The plasma frequencies are calculated from the densitydependent expressions [51]

$$
\omega_{p}^{2}=a_{g}^{2} g^{2} \frac{n_{g}}{T}+d_{q}^{2} g^{2} \frac{n_{q}}{T}
$$

for gluons and

$$
m_{f}^{2}=c_{q}^{2} g^{2} \frac{n_{q}}{T}
$$

for quarks. Here $n_{q}$ and $n_{g}$ are the quark and gluon number density, respectively. $g^{2}$ is related to the QCD running coupling constant $\alpha_{s}$ by the relation $g^{2}=4 \pi \alpha_{s}$. The coefficients $a_{g}, a_{q}, b_{q}$ are determined by demanding that as $T \rightarrow \infty, \omega_{p}$ and $m_{f}$ both go to the corresponding perturbative results. The motivation for choosing such an expression for plasma frequency is that the plasma frequency for electron-positron plasma is known to be proportional to $n / T$ in the relativistic limit $[53,54]$. Since the thermal masses appear in the expression for the density, we need to solve the density equation selfconsistently to obtain the thermal mass. The thermal mass, in turn, may be used to evaluate the thermodynamic quantities of interest. The results obtained have shown a good fit with lattice data even at temperatures near $T_{c}$ [52].

In the presence of magnetic fields, the energy eigenvalue values are given as Landau levels,

$$
E_{j}=\sqrt{m^{2}+k_{z}^{2}+2 j q_{f}|e B|},
$$

and the momentum integral is modified as [55-59]

$$
\int \frac{d^{3} k}{(2 \pi)^{3}} \rightarrow \frac{q_{f}|e B|}{2 \pi} \sum_{j=0}^{\infty} \int \frac{d k_{z}}{2 \pi}\left(2-\delta_{0 j}\right),
$$

where $f$ is the flavor index, and $q_{f}$ is the absolute value of the electric charge. The above equations modify the expression for number density. The thermomagnetic mass for quarks is obtained by using the modified equation for number density in Eq. (3) and solving the resulting equation self-consistently [45].

The expression for the number density of gluons remains unchanged in the presence of magnetic fields as gluons are chargeless and the thermomagnetic mass for gluons is obtained by solving Eq. (2) in a self-consistent manner. Note that even though the expression for gluon density remains unchanged in the presence of magnetic fields, gluons acquire a thermomagnetic mass through the quark number density. Using the thermomagnetic mass, we can obtain the thermodynamics and study the thermomagnetic properties of magnetized QGP.

We focus primarily on the qualitative thermomagnetic behavior of magnetized QGP. So, the inclusion of the effects of dynamically generated anomalous magnetic moments (AMMs) [60], as done in Refs. [61,62], is out of the scope of our work. Moreover, it has been demonstrated in Ref. [63] that the effect of AMMs is not significant when it comes to the calculation of thermodynamic quantities of charged fermions. In the self-consistent quasiparticle model, the thermomagnetic mass is obtained from the density-dependent expression for plasma frequency. It follows that the contribution from AMMs to the thermomagnetic mass is negligible. Since all quantities in this work, including the Debye mass in Sec. VII, are expressed in terms of the thermomagnetic mass, it is safe to say that the exclusion of AMMs in our work does not affect the results in any significant manner.

\section{Thermomagnetic coupling}

The only ingredient we need in order to make calculations is a thermomagnetic coupling, a coupling that incorporates the effect of both temperature and magnetic fields. The thermomagnetic coupling is used in the calculation of the thermomagnetic masses from Eqs. (2) and (3). To this end, throughout this work, we make use of the one-loop running coupling constant that evolves with both the momentum transfer and the magnetic field [64] as

$$
\alpha_{s}\left(\Lambda^{2},|e B|\right)=\frac{\alpha_{s}\left(\Lambda^{2}\right)}{1+b_{1} \alpha_{s}\left(\Lambda^{2}\right) \ln \left(\frac{\Lambda^{2}}{\Lambda^{2}+|e B|}\right)} .
$$

The one-loop running coupling in the absence of a magnetic field at the renormalization scale is given by

$$
\alpha_{s}\left(\Lambda^{2}\right)=\frac{1}{b_{1} \ln \left(\Lambda^{2} / \Lambda_{\overline{M S}}^{2}\right)},
$$

where $b_{1}=\left(11 N_{c}-2 N_{f}\right) / 12 \pi$ and following [8], $\overline{M S}=$ $0.176 \mathrm{GeV}$ at $\alpha_{s}(1.5 \mathrm{GeV})=0.326$ for $N_{f}=3$.

It is to be noted that the above thermomagnetic coupling has been obtained using the lowest Landau level approximation suitable in a strong magnetic field limit $\left(e B \gg T^{2}\right)$. As explained in Ref. [65], in this limit, the coupling is split into terms dependent on the momentum parallel and perpendicular to the magnetic field separately. The coupling dependent on the transverse momentum does not depend on the magnetic field at all. We are interested in how the system responds to magnetic fields, and so we make use of the longitudinal part of the coupling constant. The coupling is obtained in the one-loop order, and so this may be appropriate only at high temperatures. We use this coupling as an approximation, and so our results are bound to be qualitative. A two-loop thermomagnetic coupling, which includes the contribution from higher Landau levels, is expected to give quantitatively reliable results. 


\section{THERMODYNAMICS OF (2+1)-FLAVOR QGP IN THE PRESENCE OF MAGNETIC FIELDS}

In this section, we study the thermodynamics of $(2+1)$ flavor QGP, focusing only on the thermomagnetic correction. We will discuss the pure-field contributions in Sec. VI.

\section{A. Thermodynamic pressure}

For quarks, the grand canonical potential, within the selfconsistent quasiparticle model, is

$$
\begin{aligned}
\frac{\Phi_{q}}{V}= & -P_{q}=-T \frac{g_{f} q_{f}|e B|}{2 \pi^{2}} \sum_{l=1}^{\infty}(-1)^{l-1} \sum_{j=0}^{\infty}\left(2-\delta_{0 j}\right) \\
& \times\left[\frac{T}{l^{2}} \frac{m_{q_{j}} l}{T} K_{1}\left(\frac{m_{q_{j}} l}{T}\right)\right. \\
& \left.+\int_{T_{0}}^{T} \frac{d \tau}{\tau} m_{q_{j}} \frac{\partial m_{q_{j}}}{\partial \tau} K_{0}\left(\frac{m_{q_{j}} l}{\tau}\right)\right],
\end{aligned}
$$

where

$$
m_{q_{j}}=\sqrt{m_{q}^{2}+2 j\left|q_{f} e B\right|} .
$$

Here we have taken $\mu=0$. Note that in the self-consistent quasiparticle model, the grand canonical potential is not equal to $-K T \log _{10} \mathcal{Z}$, where $\mathcal{Z}$ is the grand partition function, due to the temperature dependence of masses. This is the reason why the expressions for pressure in Eqs. (8) and (10) do not match the corresponding expressions for an ideal gas even though in the quasiparticle model the system is considered as an ideal gas with temperature-dependent masses. There is an extra term that ensures thermodynamic consistency, as shown in Ref. [48]. The temperature dependence of mass in quasiparticle models has led to a great deal of discussion about the thermodynamic inconsistency problem and introduction of additional terms like $B(T)$ whose physical meaning is not obvious $[66,67]$. The self-consistent quasiparticle model avoids this problem by starting with the expressions for energy density and number density and calculating everything else from them.

The contribution from gluons is

$$
\begin{aligned}
\frac{\Phi_{g}}{V}= & -P_{g}=-T \frac{g_{f}}{2 \pi^{2}} \sum_{l=1}^{\infty} \frac{1}{l^{4}}\left[T^{3}\left(\frac{m_{g} l}{T}\right)^{2} K_{2}\left(\frac{m_{g} l}{T}\right)\right. \\
& \left.+\int_{T_{0}}^{T} \frac{d \tau}{m_{g}} \tau^{3} \frac{\partial m_{g}}{\partial \tau}\left(\frac{m_{g} l}{\tau}\right)^{3} K_{1}\left(\frac{m_{g} l}{\tau}\right)\right] .
\end{aligned}
$$

Here, $T_{0}$ is some reference temperature, suitably chosen.

\section{B. Velocity of sound}

The velocity of sound is a fundamental quantity that is used in the description of hot QCD medium. The velocity of sound squared $c_{s}^{2}$ is given by

$$
c_{s}^{2}=\frac{\partial P}{\partial \epsilon}=\frac{d P / d T}{d \epsilon / d T},
$$

where $\epsilon$ is the energy density which can be obtained from pressure using the thermodynamic relation,

$$
\epsilon=T \frac{\partial P}{\partial T}-P .
$$

The speed of sound in magnetized QGP medium can be calculated using these equations.

\section{MAGNETIZATION}

Magnetization can be obtained from the grand canonical potential $\Phi$ :

$$
\mathcal{M}=-\frac{1}{V} \frac{\partial \Phi}{\partial(e B)} .
$$

We confine our calculation to the region where $e B$ is greater than zero. Note that the equation for magnetization in the selfconsistent quasiparticle model is not related to the partition function as in Ref. [68]. This is because of the additional terms in Eqs. (8) and (10), which ensure thermodynamic consistency.

Using Eqs. (8) and (13), we get, for quarks,

$$
\begin{aligned}
\mathcal{M}_{q}= & \frac{T g_{f} q_{f}}{2 \pi^{2}} \sum_{l=1}^{\infty}(-1)^{l} \sum_{j}^{\infty}\left(2-\delta_{0 j}\right)\left(\left[\frac{T}{l^{2}}\left(\frac{m_{q j} l}{T}\right) K_{1}\left(\frac{m_{q j} l}{T}\right)\right.\right. \\
& \left.+\int_{T_{0}}^{T} \frac{d \tau}{\tau} m_{q_{j}} \frac{\partial m_{q_{j}}}{\partial \tau} K_{0}\left(\frac{m_{q_{j}} l}{\tau}\right)\right] \\
& -e B\left\{\frac{T}{l^{2}}\left(\frac{m_{q j} l}{T}\right) K_{0}\left(\frac{m_{q j} l}{T}\right) \frac{\partial}{\partial(e B)}\left(\frac{m_{q j} l}{T}\right)\right. \\
& \left.\left.-\frac{\partial}{\partial(e B)}\left[\int_{T_{0}}^{T} \frac{d \tau}{\tau} m_{q_{j}} \frac{\partial m_{q_{j}}}{\partial \tau} K_{0}\left(\frac{m_{q_{j}} l}{\tau}\right)\right]\right\}\right) .
\end{aligned}
$$

In a similar manner, we obtain the expression for magnetization of gluons from Eq. (10) as

$$
\begin{aligned}
\mathcal{M}_{g}= & \frac{T g_{f}}{2 \pi^{2}} \sum_{l=1}^{\infty}\left[\frac{\partial}{\partial(e B)} \int_{T_{0}}^{T} \frac{d \tau}{m_{g}} \tau^{3} \frac{\partial m_{g}}{\partial \tau}\left(\frac{m_{g} l}{\tau}\right)^{3}\right. \\
& \times K_{1}\left(\frac{m_{g} l}{\tau}\right)-T^{3}\left(\frac{m_{g} l}{T}\right)^{2} \\
& \left.\times K_{1}\left(\frac{m_{g} l}{T}\right) \frac{\partial}{\partial(e B)}\left(\frac{m_{g} l}{T}\right)\right] .
\end{aligned}
$$

By obtaining the magnetization using the above equations, we can go on to study the pressure anisotropy of QGP in the presence of magnetic fields.

\section{PRESSURE ANISOTROPY}

There has been some discussion in the literature regarding the existence of a pressure anisotropy, and it has been suggested that the anisotropy is scheme dependent [68-76]. In the $\phi$ scheme, the presence of magnetic fields breaks rotational symmetry due to the magnetization of the system in the direction of the magnetic field, resulting in a pressure anisotropy. Thus, in this scheme, the pressure has a transverse component different from the longitudinal component. 
The transverse pressure is related to the longitudinal pressure as

$$
P_{T}=P-e B \cdot \mathcal{M}
$$

Using Eqs. (8) and (14) the contribution from quarks to transverse pressure becomes

$$
\begin{aligned}
\frac{\left(P_{T}\right)_{q}}{T}= & \frac{g_{f} q_{f}(e B)^{2}}{2 \pi^{2}} \sum_{l=1}^{\infty}(-1)^{l} \sum_{j}^{\infty}\left(2-\delta_{0 j}\right) \\
& \times\left\{\frac{T}{l^{2}}\left(\frac{m_{q j} l}{T}\right) K_{0}\left(\frac{m_{q j} l}{T}\right) \frac{\partial}{\partial(e B)}\left(\frac{m_{q j} l}{T}\right)\right. \\
& \left.-\frac{\partial}{\partial(e B)}\left[\int_{T_{0}}^{T} \frac{d \tau}{\tau} m_{q_{j}} \frac{\partial m_{q_{j}}}{\partial \tau} K_{0}\left(\frac{m_{q_{j}} l}{\tau}\right)\right]\right\} .
\end{aligned}
$$

Similarly, for gluons,

$$
\begin{aligned}
\frac{\left(P_{T}\right)_{g}}{T}= & \frac{g_{f}}{2 \pi^{2}} \sum_{l=1}^{\infty} \frac{1}{l^{4}}\left\{T^{3}\left(\frac{m_{g} l}{T}\right)^{2} K_{2}\left(\frac{m_{g} l}{T}\right)\right. \\
& +\int_{T_{0}}^{T} \frac{d \tau}{m_{g}} \tau^{3} \frac{\partial m_{g}}{\partial \tau}\left(\frac{m_{g} l}{\tau}\right)^{3} K_{1}\left(\frac{m_{g} l}{\tau}\right) \\
& -e B\left[\frac{\partial}{\partial(e B)} \int_{T_{0}}^{T} \frac{d \tau}{m_{g}} \tau^{3} \frac{\partial m_{g}}{\partial \tau}\left(\frac{m_{g} l}{\tau}\right)^{3} K_{1}\left(\frac{m_{g} l}{\tau}\right)\right. \\
& \left.\left.-T^{3}\left(\frac{m_{g} l}{T}\right)^{2} K_{1}\left(\frac{m_{g} l}{T}\right) \frac{\partial}{\partial(e B)}\left(\frac{m_{g} l}{T}\right)\right]\right\} .
\end{aligned}
$$

\section{PURE-FIELD CONTRIBUTION}

So far we concentrated only on the contributions to the thermodynamic quantities from quarks and gluons. The total pressure of magnetized QGP involves one other contribution, namely, the pure-field or Maxwell contribution [69,75,77-79]. These contributions are again different in the parallel and perpendicular directions as the magnetic field breaks rotational symmetry. The total pressure in the transverse direction is

$$
\begin{aligned}
P_{T}^{\text {total }} & =P_{T}+\left(P_{T}\right)_{m} \\
& =P_{T}+\frac{B^{2}}{2} .
\end{aligned}
$$

The total pressure in the longitudinal direction is given by

$$
\begin{aligned}
P^{\text {total }} & =P+P_{m} \\
& =P-\frac{B^{2}}{2},
\end{aligned}
$$

where $\left(P_{T}\right)_{m}$ and $P_{m}$ are the pure-field contributions in the transverse and longitudinal directions, respectively. Using these we can calculate the total longitudinal and transverse pressures.

\section{LONGITUDINAL DEBYE SCREENING MASS}

At the leading order, Debye screening mass parametrizes the dynamically generated screening of chromoelectric fields, due to the strong interactions of hot quantum chromodynamics [80]. The ability of QGP to shield out the chromoelectric potential can be measured in terms of the Debye screening length, which is the inverse of the Debye mass $\left(m_{D}\right)$. Calculations of the higher-order contributions to the Debye screening $[81,82]$ are beyond the scope of this work. The presence of an external magnetic field causes an anisotropy, and we study the Debye mass in the longitudinal direction.

The conventional definition for Debye mass can be obtained either from the small momentum limit of the gluon self-energy [83-87] or the semiclassical transport theory $[44,88,89]$.

In the zero magnetic field case, the Debye mass can be defined as $[44,85,90]$

$$
m_{D}^{2}=-g_{n} g^{2} \int \frac{d^{3} k}{(2 \pi)^{3}} \frac{\partial}{\partial \omega_{k}} f\left(\omega_{k}\right),
$$

where $f\left(\omega_{k}\right)$ are the quasi-gluon, quasi-quark-antiquark distribution functions with

$$
\omega_{k}=\sqrt{m(T)^{2}+k^{2}},
$$

and $g_{n}$ is the degeneracy factor. In the self-consistent quasiparticle model, all the medium effects are captured by the thermal masses of the quasiparticles $m(T)$. The distribution functions (in zero chemical potential) are

$$
f_{g}\left(\omega_{k}\right)=\frac{1}{e^{\beta \omega_{k}}-1} \quad \text { and } \quad f_{q}\left(\omega_{k}^{f}\right)=\frac{1}{e^{\beta \omega_{k}^{f}}+1}
$$

for gluons and quark-antiquark flavor $f$, respectively. Hereafter we shall drop the flavor index on the quasiparticle mass and energies of quarks, to avoid cluttering.

The gluonic contribution to the Debye mass in zero magnetic field is obtained using Eqs. (21) and (22), the first relation in Eq. (23), and $g_{n}=2 N_{c}$ as

$$
m_{D g}^{2}=\frac{N_{c} g^{2}}{\pi^{2} T} \int_{0}^{\infty} d k k^{2} \frac{e^{-\beta \sqrt{k^{2}+m_{g}^{2}}}}{\left(1-e^{-\beta \sqrt{k^{2}+m_{g}^{2}}}\right)^{2}},
$$

which simplifies to

$$
m_{D g}^{2}=\frac{N_{c} g^{2} T^{2}}{\pi^{2}}\left[\sum_{l=1}^{\infty} \frac{1}{l^{2}}\left(\frac{l m_{g}}{T}\right)^{2} K_{2}\left(\frac{l m_{g}}{T}\right)\right] .
$$

The gluon Debye mass is associated with the gluon and ghost loops contribution to the gluon self-energy. In the presence of magnetic fields, the expression of Debye mass for gluons remains the same as the magnetic fields do not change the ghost and gluon loops [8]. The expression for gluon Debye mass in the self-consistent quasiparticle model also remains the same as Eq. (25). However, the thermomagnetic mass of gluons, $m_{g}$, depends on magnetic fields through the quark contribution to the gluon plasma frequency. The gluon plasma frequency in Eq. (2) is defined in such a way that it approaches the corresponding values in perturbative QCD at high temperatures, ensuring consistency. So we can consider the dependence of the gluon Debye mass on magnetic fields, through the quark plasma frequency, as a correction to the perturbative result due to medium effects caused by the strong interaction. 


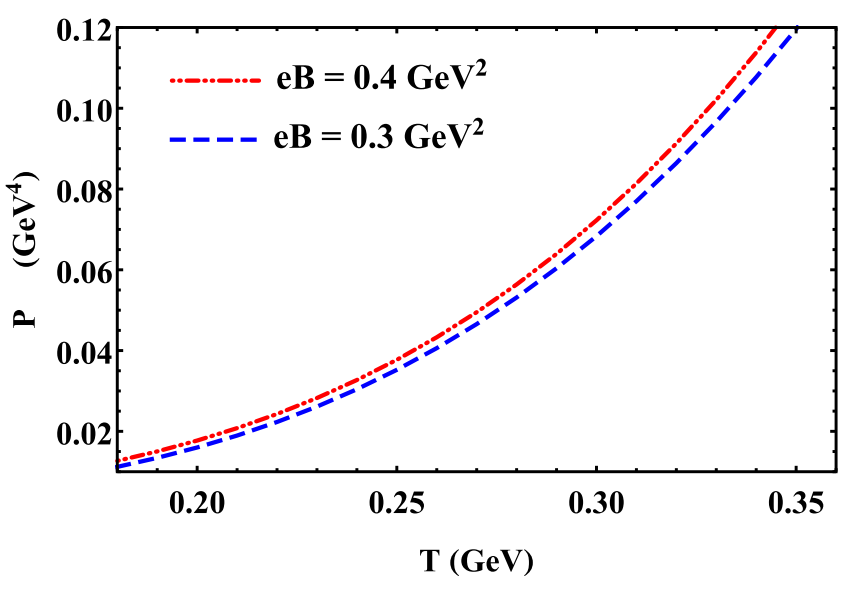

FIG. 1. Thermodynamic pressure as a function of temperature for different magnetic fields.

At zero magnetic field, Eq. (21), with the quark distribution function in Eq. (23), can also be written as

$$
m_{D q}^{2}=\frac{g^{2}}{T} g_{n} \int \frac{d^{3} k}{(2 \pi)^{3}} f\left(\omega_{k}\right)\left[1-f\left(\omega_{k}\right)\right] .
$$

With $g_{n}=2 \times 2 \times N_{c}$ (quark-antiquark, spin, and color), and for a single quark flavor,

$$
m_{D q}^{2}=\frac{4 N_{c} g^{2}}{T} \int \frac{d^{3} k}{(2 \pi)^{3}} \frac{e^{-\beta \sqrt{k^{2}+m_{q}^{2}}}}{\left(1+e^{-\beta \sqrt{k^{2}+m_{q}^{2}}} .\right)^{2}},
$$

which simplifies to

$$
m_{D q}^{2}=\frac{2 N_{c} g^{2} T^{2}}{\pi^{2}} \sum_{l=1}^{\infty} \frac{(-1)^{l-1}}{l^{2}}\left(\frac{l m_{q}}{T}\right)^{2} K_{2}\left(\frac{l m_{q}}{T}\right) .
$$

The Debye mass for quarks is associated with the quark loop contribution to the gluon self-energy. This changes in the presence of external magnetic fields. The expression of Debye mass for quarks in the presence of magnetic fields can be obtained by replacing the thermal masses by thermomagnetic masses, changing the dispersion relation in accordance with Eq. (4), and modifying the momentum integration according to Eq. (5) in Eq. (26). This gives [85]

$$
m_{D q}^{2}(e B)=\frac{\left|q_{f} e B\right| g^{2}}{2 \pi^{2}} \sum_{j=0}^{\infty} g_{j} \int_{0}^{\infty} \frac{d k_{z}}{T} f_{q}\left(\omega_{k_{z}}^{j}\right)\left[1-f_{q}\left(\omega_{k_{z}}^{j}\right)\right],
$$

where

$$
f_{q}\left(\omega_{k_{z}}^{j}\right)=\frac{1}{e^{\beta \omega_{k_{z}}^{j}}+1}
$$

and

$$
\omega_{k_{z}}^{j}=\sqrt{k_{z}^{2}+m_{q_{j}}^{2}},
$$

with $m_{q_{j}}$ given in Eq. (9). $g_{j}$ in Eq. (29) is the degeneracy of the $j^{\text {th }}$ Landau level and is dependent on the Landau level index $j$. Along with these and adding up the contributions

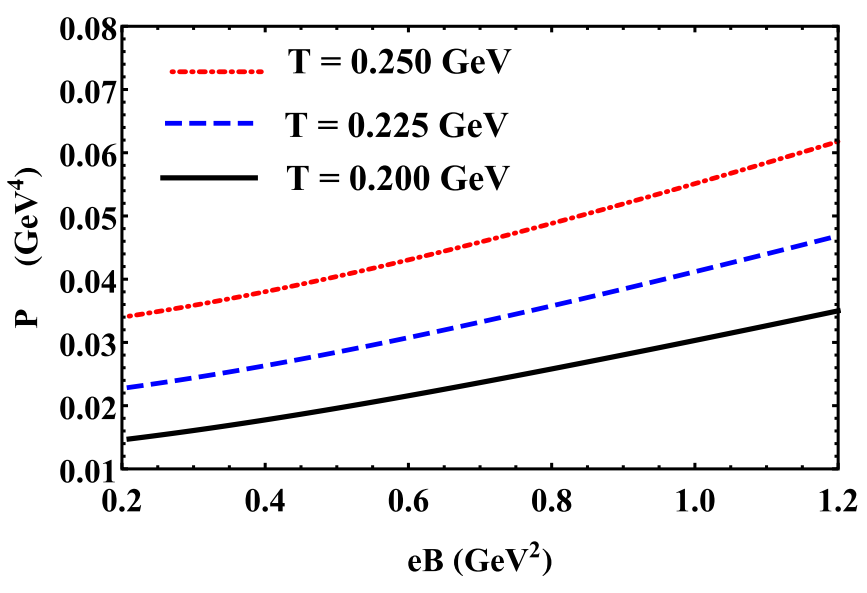

FIG. 2. Thermodynamic pressure for different temperatures as a function of magnetic field.

from all flavors, we get from Eq. (29)

$$
m_{D q}^{2}=\sum_{f} \frac{\left|q_{f} e B\right| g^{2}}{2 \pi^{2}} \sum_{j=0}^{\infty} g_{j} \int_{0}^{\infty} \frac{d k_{z}}{T} \frac{e^{-\beta \sqrt{k_{z}^{2}+m_{q_{j}}^{2}}}}{\left(1+e^{-\beta \sqrt{k_{z}^{2}+m_{q_{j}}^{2}}}\right)^{2}} .
$$

With $g_{j}=2 N_{c} \times\left(2-\delta_{0 j}\right)$ [85], Eq. (32) simplifies to

$$
\begin{aligned}
m_{D q}^{2}(e B)= & \frac{N_{c} g^{2}}{\pi^{2}} \sum_{f}\left|q_{f} e B\right| \sum_{l=1}^{\infty}(-1)^{l-1} \sum_{j=0}^{\infty}\left(2-\delta_{0 j}\right) \\
& \times\left(\frac{l m_{q_{j}}}{T}\right) K_{1}\left(\frac{l m_{q_{j}}}{T}\right) .
\end{aligned}
$$

\section{RESULTS AND DISCUSSION}

We have plotted the variation of longitudinal pressure with temperature for different magnetic fields in Fig. 1. Figure 2 shows the variation of pressure with the magnetic field for different temperatures. The increase in pressure with a magnetic field at a given temperature, as seen in our equation of state, is consistent with lattice QCD results [68], perturbative QCD

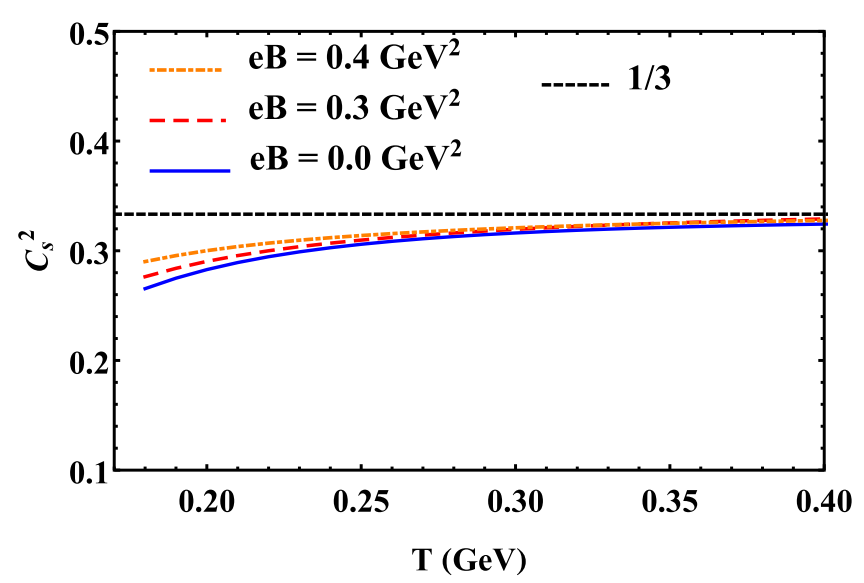

FIG. 3. Velocity of sound as a function of temperature for different values of magnetic fields. 


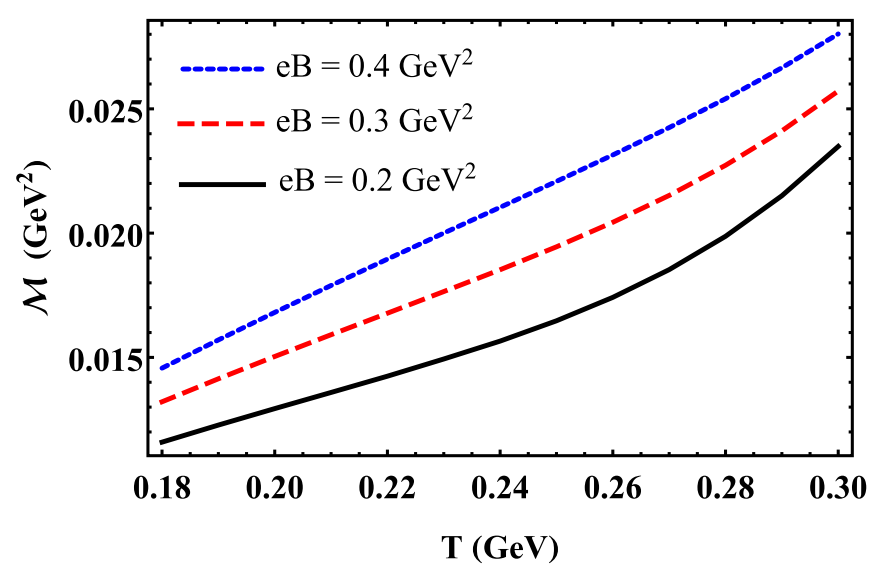

FIG. 4. Magnetization for different magnetic fields as a function of temperature.

results [8], and other works [44]. At this point, we do not make a quantitative comparison with the lattice data because the coupling constant used here is calculated in the lowest Landau level (LLL) approximation. The one-loop thermomagnetic coupling we used may be reliable at high temperatures only.

In Fig. 3 we have plotted $C_{s}^{2}$ as a function of temperature, for different magnetic field values. The speed of sound is seen to reach the Stefan-Boltzmann limit of $1 / 3$, asymptotically. This behavior is consistent with the behavior of $P / \epsilon$ in lattice QCD results [68] and with the behavior of sound velocity in Ref. [44].

The variation of magnetization with temperature for different magnetic fields is plotted in Fig. 4. We see that the magnetization has a positive value for all values of temperature above $T_{c}$. This shows that QGP has a paramagnetic nature.

In Fig. 5, we have plotted the variation of magnetization with the magnetic field for different temperatures. It is seen that the magnetization increases with the magnetic field. The behavior of magnetization of QGP, as seen in our work, is qualitatively consistent with lattice QCD results [91] and with results from hard thermal loop (HTL) perturbation theory [8]. We have included higher Landau levels, whereas in Ref. [8]

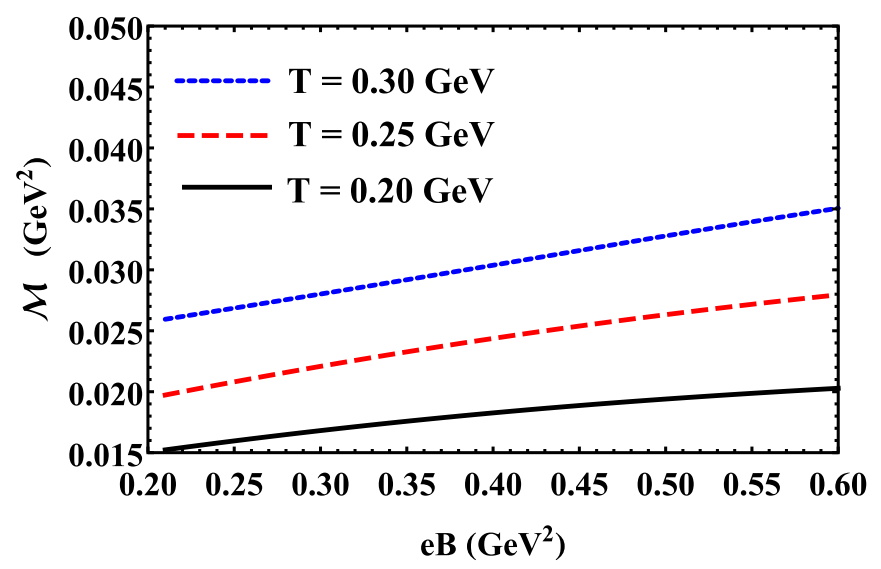

FIG. 5. Magnetization for different temperatures as a function of magnetic field.

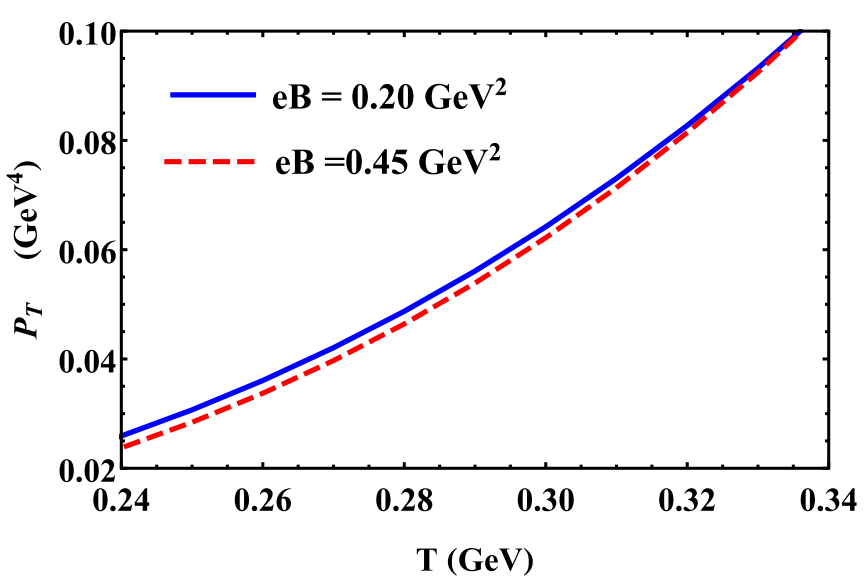

FIG. 6. Transverse pressure for different magnetic fields as a function of temperature.

the lowest Landau level approximation has been used. We see that in our model, the contribution from higher Landau levels cannot be neglected.

In Fig. 6, we have plotted the variation of transverse pressure with temperature for different magnetic fields. In Fig. 7, we show the variation of transverse pressure with magnetic fields for different temperatures. Since magnetization increases with temperature, the transverse pressure tends to decrease with an increase in the magnetic field. This behavior, too, is qualitatively consistent with the perturbative QCD results from [8], and the lattice QCD results from [68,91].

Including the Maxwell contribution to the total pressure, we see that the parallel or longitudinal pressure decreases and the transverse pressure increases with increase in the magnetic field strength. The behavior of the total longitudinal and transverse pressure is plotted in Figs. 8-11. Figure 8 shows that for a given magnetic field, the total longitudinal pressure increases with temperature. Toward the lower values of temperature the value of total longitudinal pressure is negative. Figure 9 shows that the total longitudinal pressure decreases and becomes negative as the magnetic field strength is increased. The negative value of total longitudinal pressure

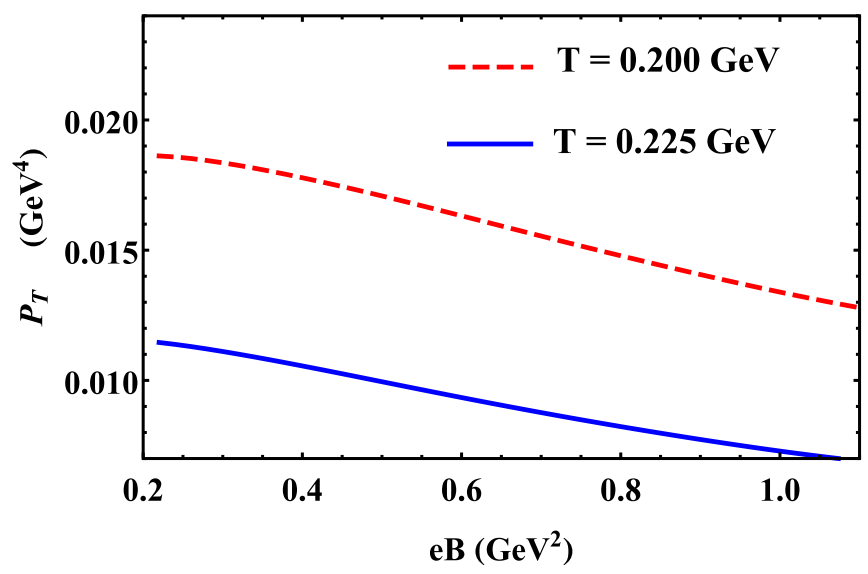

FIG. 7. Transverse pressure for different temperatures as a function of magnetic field. 


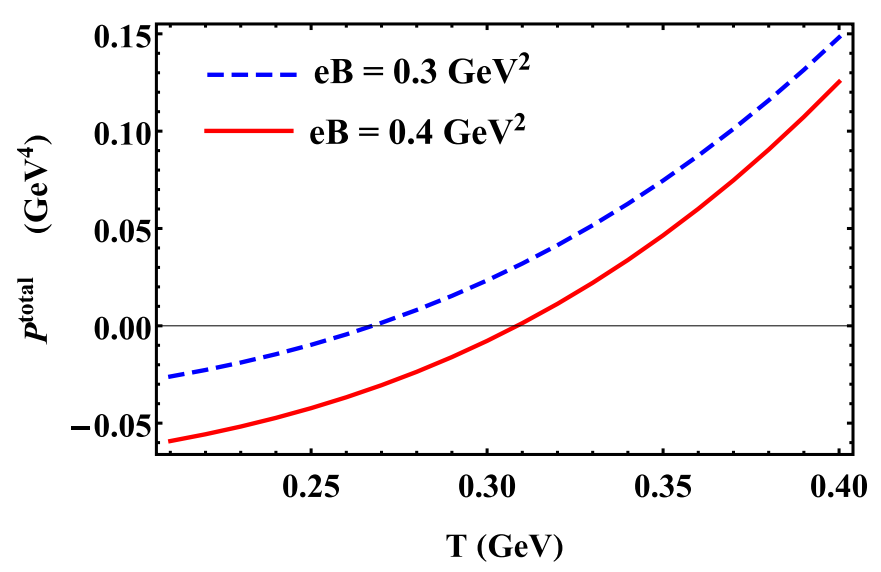

FIG. 8. Variation of total longitudinal pressure $\left(P+P_{m}\right)$ with temperature for different magnetic fields.

can produce instabilities to the system and this aspect deserves to be studied carefully. The total transverse pressure (Fig. 11) increases with increase in magnetic field and remains positive.

We study the screening effect in magnetized QGP using our model by calculating the Debye mass. At $B=0$, the Debye mass increases with temperature. We have plotted the variation of Debye mass with temperature for different magnetic fields in Fig. 12. The Debye screening mass variation with magnetic fields is plotted for different temperatures in Fig. 13. The enhancement of Debye screening mass in the presence of external magnetic field agrees with the finding in Ref. [82] using lattice QCD simulations, and in Refs. [14,26,92] using perturbative calculations. Similar results are also obtained in Refs. [44,85].

In Fig. 14, we plot our result for the contribution of quarks to the Debye mass along with the corresponding result from perturbative quantum chromodynamics (pQCD). The expression for the Debye mass of quarks obtained from the gluon self-energy tensor in the strong magnetic field limit and with

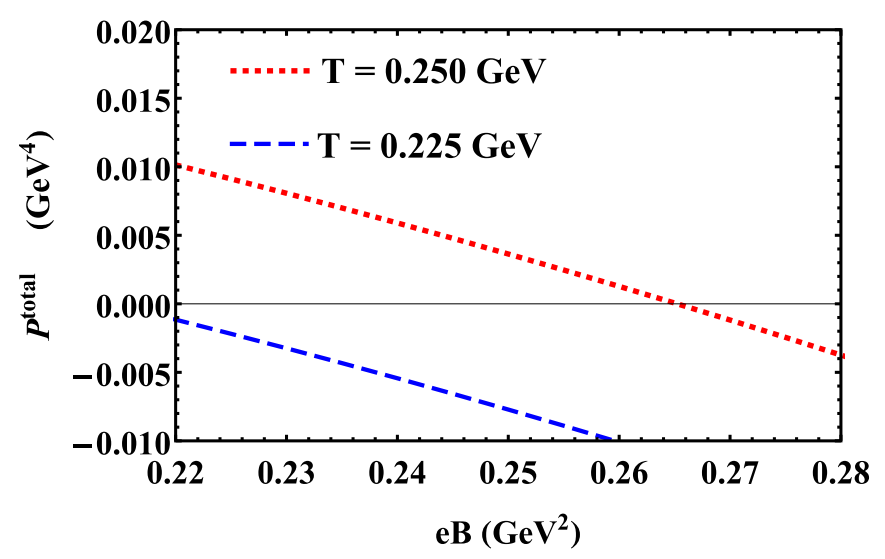

FIG. 9. Variation of total longitudinal pressure with magnetic field for different temperatures.

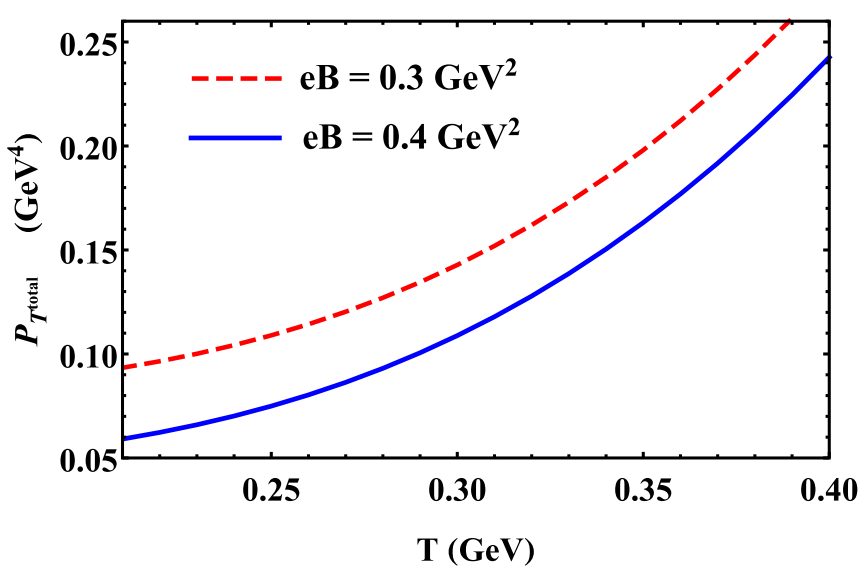

FIG. 10. Variation of total transverse pressure $\left[P_{T}+\left(P_{T}\right)_{m}\right]$ with temperature for different magnetic fields.

massless quarks [93-95] is

$$
m_{D}^{2}=\frac{g^{2}}{4 \pi^{2}} \sum_{f}\left|q_{f} e B\right| .
$$

We have corrected for a factor of 2 overlooked in Ref. [94] as pointed out in Ref. [95]. We plot the quark Debye mass of Eq. (34) in Fig. 14, after including the appropriate degeneracy factor $g_{n}=2 N_{c}$ [85]. We have used $g^{2}=4 \pi \alpha_{s}$, with the thermomagnetic coupling $\alpha_{s}$ given in Eq. (6). The plots are denoted as "pQCD."

Along with the pQCD results in Fig. 14, we have plotted the quark Debye mass calculated using Eq. (33) in the LLL approximation and neglecting the physical quark masses $\left(m_{0}=0\right)$. The quasiparticles, however, are still massive with masses depending on temperature and magnetic fields.

At $T=0.2 \mathrm{GeV}$ our results deviate from the $\mathrm{pQCD}$ results. The deviation is expected because QGP is strongly interacting at these temperatures, and perturbative QCD may not be suitable. In our model, the strong interaction is taken into account through the temperature and magnetic field dependence of the quasiparticle masses.

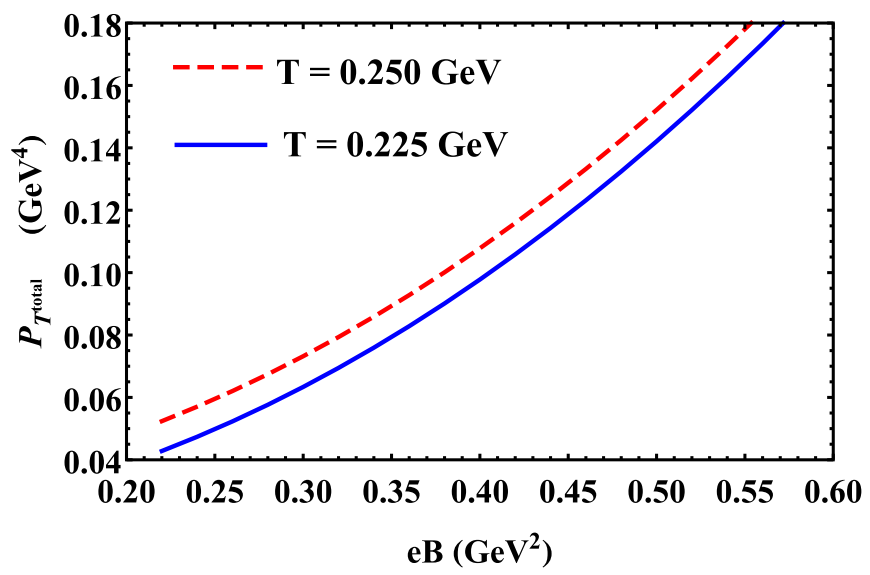

FIG. 11. Variation of total transverse pressure with magnetic field for different temperatures. 


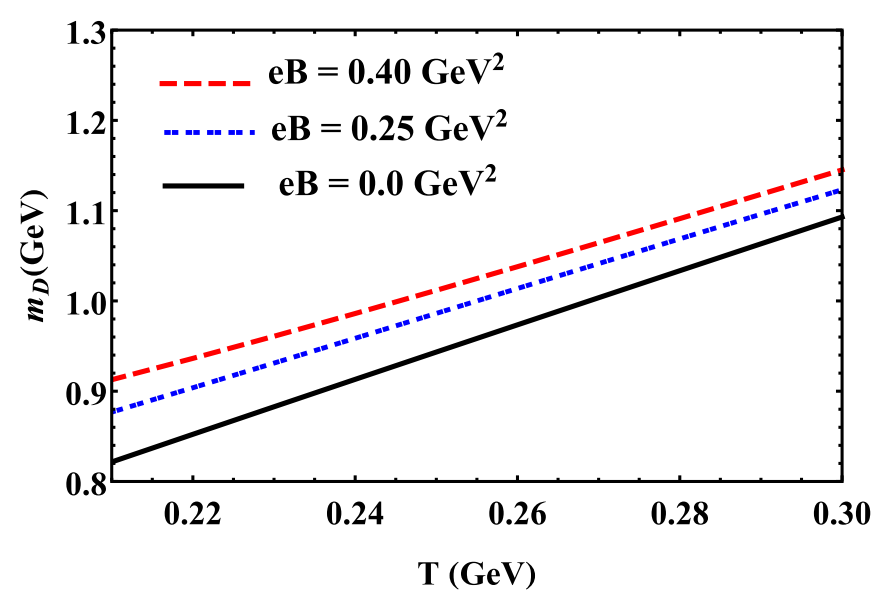

FIG. 12. Debye mass as a function of temperature for different magnetic fields.

The results from these different approaches match reasonably well at a higher temperature of $T=0.4 \mathrm{GeV}$, as shown in the plot. The agreement shows that our results approach the results obtained through a perturbative calculation at high temperatures. The proportionality constants in Eqs. (2) and (3) are obtained by demanding that the expressions for plasma frequency approach the corresponding pQCD results at high temperature. So the comparison plot shows that our approach is consistent.

The expression for Debye mass of massive quarks in Ref. [94], after correcting for the missing factor of 2 and multiplying by the degeneracy factor, is

$$
m_{D}^{2}=\frac{N_{c} g^{2}}{\pi^{2}} \sum_{f}\left|q_{f} e B\right| \int_{0}^{\infty} \frac{d k_{z}}{T} \frac{e^{\beta \sqrt{k_{z}^{2}+m_{0}^{2}}}}{\left(1+e^{\beta \sqrt{k_{z}^{2}+m_{0}^{2}}}\right)^{2}},
$$

where $m_{0}$ is the physical quark mass (we have dropped the flavor index for convenience). We note that Eq. (32), obtained from the self-consistent quasiparticle model, reduces to Eq. (35) in the LLL approximation and when quarks and gluons are considered as noninteracting $\left(m_{q_{j}}=m_{0}\right)$. Inter-

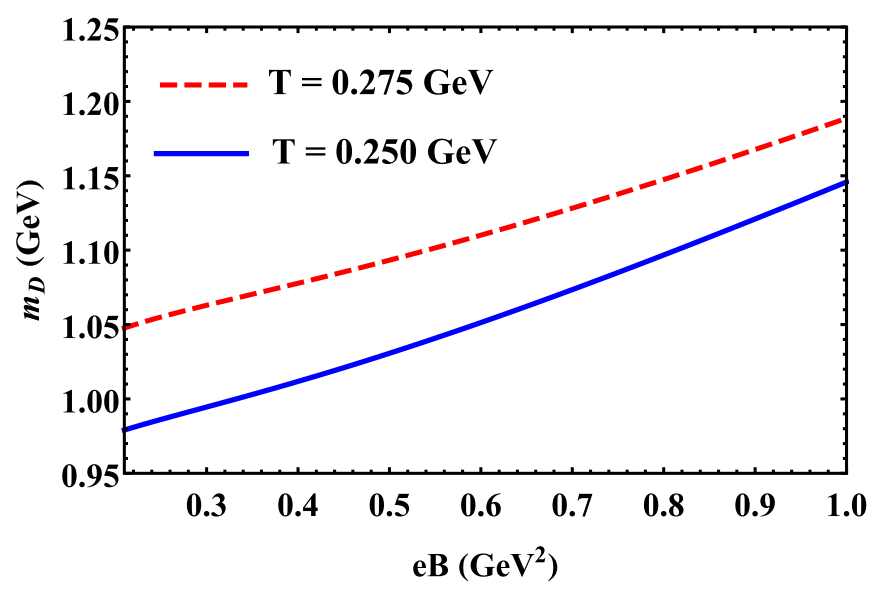

FIG. 13. Debye mass as a function of magnetic field for different temperatures.

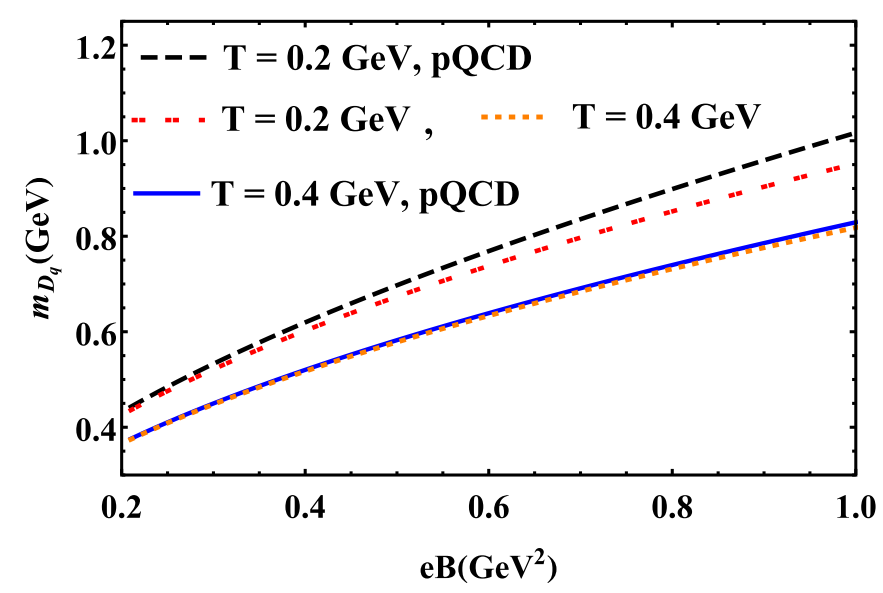

FIG. 14. Contribution of quarks to the Debye mass plotted against magnetic field compared with $\mathrm{pQCD}$ result.

acting quarks and gluons acquire thermomagnetic mass, and the stronger the interaction, the more significant the deviation from the ideal gas result.

\section{CONCLUSION}

To summarize, we made use of the extended self-consistent quasiparticle model to study thermodynamics, thermomagnetic properties, and screening behavior of magnetized $(2+1)$ flavor quark-gluon plasma.

We studied the thermodynamics of magnetized $(2+1)$ flavor QGP by calculating and plotting the pressure and sound velocity of magnetized QGP. The magnetic response of QGP was investigated by our model, and the variation of magnetization with temperature and magnetic field examined. We found that QGP has a paramagnetic nature. It has a small but positive magnetization at all temperatures above the transition temperature. We also noted that the presence of magnetization makes the system anisotropic, causing different pressures in directions parallel and perpendicular to the magnetic field. We evaluated the transverse pressure and plotted its variation with both magnetic fields and temperature. The equation of state and anisotropic pressure calculated here can be used as an input for magnetohydrodynamic calculations and analysis of the elliptic flow of QGP formed in heavy-ion collisions. We noted that taking the pure-field contribution into account, the total longitudinal pressure becomes negative, indicating instability in the system in this region. The total transverse pressure remains positive and increases with the magnetic field in the range of magnetic fields and temperatures considered in this work. Finally, we studied the screening properties of magnetized QGP by examining the behavior of Debye screening mass in the longitudinal direction. We saw that the screening mass increases with magnetic fields. Our results showed the same qualitative behavior as those obtained from lattice QCD calculations and the HTL perturbation theory approach, and those obtained using other phenomenological models. The quark contribution to Debye mass as calculated and plotted using our model agrees reasonably well with 
the corresponding results from perturbative QCD, at high temperatures.

We see that the extended quasiparticle model is quite effective in studying various thermodynamic and thermomagnetic properties of the deconfined QCD matter in the presence of magnetic fields. It is an advantage to this model that the higher Landau level contributions can be incorporated without difficulty. The present results could be improved with a two-loop order thermomagnetic coupling, which also includes the contributions from higher Landau levels.

It would be interesting to study the transport coefficients of magnetized QGP with the equation of state obtained using the extended self-consistent quasiparticle model. Another area where the model, with appropriate modifications, can be applied is QGP at finite temperature and density. We can use the model extended to nonzero chemical potential to study the interior of strongly magnetized neutron stars.

\section{ACKNOWLEDGMENTS}

S.K. thanks Manu Kurian for helpful discussions. S.K. would like to thank University Grants Commission (UGC), New Delhi, for support from a BSR-SAP fellowship [Fellowship No. 25-1/2014-15 (BSR)/7-180/2007 (BSR)] during the period of research.
[1] B. Back et al., Nucl. Phys. A 757, 28 (2005); J. Adams et al., ibid. 757, 102 (2005); K. Adcox et al., ibid. 757, 184 (2005); I. Arsene et al., ibid. 757, 1 (2005).

[2] V. V. Skokov, A. Y. Illarionov, and V. D. Toneev, Int. J. Mod. Phys. A 24, 5925 (2009).

[3] Z. Yang, Y. Chun-Bin, C. Xu, and F. Sheng-Qin, Chin. Phys. C 39, 104105 (2015).

[4] K. Tuchin, Phys. Rev. C 83, 017901 (2011).

[5] K. Marasinghe and K. Tuchin, Phys. Rev. C 84, 044908 (2011).

[6] K. Tuchin, Phys. Rev. C 82, 034904 (2010).

[7] K. Fukushima and J. M. Pawlowski, Phys. Rev. D 86, 076013 (2012).

[8] B. Karmakar, R. Ghosh, A. Bandyopadhyay, N. Haque, and M. G. Mustafa, Phys. Rev. D 99, 094002 (2019).

[9] M. Kurian and V. Chandra, Phys. Rev. D 97, 116008 (2018).

[10] M. Kurian, S. K. Das, and V. Chandra, Phys. Rev. D 100, 074003 (2019).

[11] M. Kurian and V. Chandra, Phys. Rev. D 99, 116018 (2019).

[12] A. Mukherjee, S. Ghosh, M. Mandal, S. Sarkar, and P. Roy, Phys. Rev. D 98, 056024 (2018).

[13] M. Kurian, S. Mitra, S. Ghosh, and V. Chandra, Eur. Phys. J. C 79, 134 (2019).

[14] A. Bandyopadhyay, C. A. Islam, and M. G. Mustafa, Phys. Rev. D 94, 114034 (2016).

[15] G. Endrődi, J. High Energy Phys. 04 (2013) 023.

[16] L. Levkova and C. DeTar, Phys. Rev. Lett. 112, 012002 (2014).

[17] S. Ghosh and V. Chandra, arXiv:1901.04322.

[18] V. P. Gusynin, V. A. Miransky, and I. A. Shovkovy, Phys. Rev. Lett. 73, 3499 (1994).

[19] D. Kharzeev et al., Strongly Interacting Matter in Magnetic Fields (Springer, Berlin, 2013).

[20] V. Gusynin, V. Miransky, and I. Shovkovy, Nucl. Phys. B 462, 249 (1996).

[21] K. Fukushima and Y. Hidaka, Phys. Rev. Lett. 110, 031601 (2013).

[22] K. Fukushima, D. E. Kharzeev, and H. J. Warringa, Phys. Rev. D 78, 074033 (2008).

[23] D. E. Kharzeev, L. D. McLerran, and H. J. Warringa, Nucl. Phys. A 803, 227 (2008).

[24] D. E. Kharzeev, Prog. Part. Nucl. Phys. 75, 133 (2014).

[25] S. Ghosh, A. Bandyopadhyay, R. L. S. Farias, J. Dey, and G. Krein, arXiv:1911.10005.

[26] A. Bandyopadhyay, R. L. S. Farias, B. S. Lopes, and R. O. Ramos, Phys. Rev. D 100, 076021 (2019).
[27] S. K. Das, S. Plumari, S. Chatterjee, J. Alam, F. Scardina, and V. Greco, Phys. Lett. B 768, 260 (2017).

[28] S. S. Avancini, R. L. S. Farias, N. N. Scoccola, and W. R. Tavares, Phys. Rev. D 99, 116002 (2019).

[29] R. L. S. Farias, K. P. Gomes, G. Krein, and M. B. Pinto, Phys. Rev. C 90, 025203 (2014).

[30] R. Rougemont, R. Critelli, and J. Noronha, Phys. Rev. D 93, 045013 (2016).

[31] S. Fayazbakhsh and N. Sadooghi, Phys. Rev. D 83, 025026 (2011).

[32] S. Fayazbakhsh and N. Sadooghi, Phys. Rev. D 82, 045010 (2010).

[33] B. Singh, L. Thakur, and H. Mishra, Phys. Rev. D 97, 096011 (2018).

[34] S. Acharya et al., Phys. Rev. Lett. 125, 022301 (2020).

[35] J. Adam et al., Phys. Rev. Lett. 123, 162301 (2019).

[36] S. Bhadury, M. Kurian, V. Chandra, and A. Jaiswal, J. Phys. G: Nucl. Part. Phys. 47, 085108 (2020).

[37] D. Grasso and H. R. Rubinstein, Phys. Rep. 348, 163 (2001)

[38] R. C. Duncan and C. Thompson, Astrophys. J. 392, L9 (1992).

[39] A. Broderick, M. Prakash, and J. M. Lattimer, Astrophys. J. 537, 351 (2000).

[40] C. Y. Cardall, M. Prakash, and J. M. Lattimer, Astrophys. J. 554, 322 (2001).

[41] A. Rabhi, C. Providência, and J. D. Providência, J. Phys. G: Nucl. Part. Phys. 35, 125201 (2008).

[42] A. Rabhi, P. K. Panda, and C. Providência, Phys. Rev. C 84, 035803 (2011).

[43] S. S. Avancini, V. Dexheimer, R. L. S. Farias, and V. S. Timóteo, Phys. Rev. C 97, 035207 (2018).

[44] M. Kurian and V. Chandra, Phys. Rev. D 96, 114026 (2017).

[45] S. Koothottil and V. M. Bannur, Phys. Rev. C 99, 035210 (2019).

[46] R. L. S. Farias, V. S. Timóteo, S. S. Avancini, M. B. Pinto, and G. Krein, Eur. Phys. J. A 53, 101 (2017).

[47] V. M. Bannur, Phys. Rev. C 75, 044905 (2007).

[48] V. M. Bannur, Phys. Lett. B 647, 271 (2007).

[49] V. M. Bannur, J. High Energy Phys. 09 (2007) 046.

[50] V. M. Bannur, Eur. Phys. J. C 50, 629 (2007).

[51] V. M. Bannur, Phys. Rev. C 78, 045206 (2008).

[52] V. M. Bannur, Int. J. Mod. Phys. E 21, 1250090 (2012).

[53] M. V. Medvedev, Phys. Rev. E 59, R4766 (1999). 
[54] V. M. Bannur, Phys. Rev. E 73, 067401 (2006).

[55] E. S. Fraga and L. F. Palhares, Phys. Rev. D 86, 016008 (2012).

[56] A. J. Mizher, M. N. Chernodub, and E. S. Fraga, Phys. Rev. D 82, 105016 (2010).

[57] S. Chakrabarty, Phys. Rev. D 54, 1306 (1996).

[58] F. Bruckmann, G. Endrődi, M. Giordano, S. D. Katz, T. G. Kovács, F. Pittler, and J. Wellnhofer, Phys. Rev. D 96, 074506 (2017).

[59] A. N. Tawfik, J. Phys.: Conf. Ser. 668, 012082 (2016).

[60] E. J. Ferrer and V. de la Incera, Nucl. Phys. B 824, 217 (2010).

[61] N. Chaudhuri, S. Ghosh, S. Sarkar, and P. Roy, arXiv:2003.05692.

[62] M. Strickland, V. Dexheimer, and D. P. Menezes, Phys. Rev. D 86, 125032 (2012).

[63] E. J. Ferrer, V. de la Incera, D. M. Paret, A. P. Martínez, and A. Sanchez, Phys. Rev. D 91, 085041 (2015).

[64] A. Ayala, C. A. Dominguez, S. Hernandez-Ortiz, L. A. Hernandez, M. Loewe, D. M. Paret, and R. Zamora, Phys. Rev. D 98, 031501 (2018).

[65] E. J. Ferrer, V. de la Incera, and X. J. Wen, Phys. Rev. D 91, 054006 (2015).

[66] M. I. Gorenstein and S. N. Yang, Phys. Rev. D 52, 5206 (1995).

[67] F. Gardim and F. Steffens, Nucl. Phys. A 797, 50 (2007).

[68] G. S. Bali, F. Bruckmann, G. Endrödi, S. D. Katz, and A. Schäfer, J. High Energy Phys. 08 (2014) 177.

[69] E. J. Ferrer, V. de la Incera, J. P. Keith, I. Portillo, and P. L. Springsteen, Phys. Rev. C 82, 065802 (2010).

[70] K. Kohri, S. Yamada, and S. Nagataki, Astropart. Phys. 21, 433 (2004).

[71] A. P. Martínez, H. P. Rojas, and H. J. M. Cuesta, Eur. Phys. J. C 29, 111 (2003).

[72] M. Chaichian, S. S. Masood, C. Montonen, A. Perez Martínez, and H. Perez Rojas, Phys. Rev. Lett. 84, 5261 (2000).

[73] A. Y. Potekhin and D. G. Yakovlev, Phys. Rev. C 85, 039801 (2012).
[74] E. J. Ferrer, V. de la Incera, J. P. Keith, I. Portillo, and P. L. Springsteen, Phys. Rev. C 85, 039802 (2012).

[75] V. Dexheimer, D. P. Menezes, and M. Strickland, J. Phys. G: Nucl. Part. Phys. 41, 015203 (2013).

[76] G. S. Bali, F. Bruckmann, G. Endrődi, F. Gruber, and A. Schäfer, J. High Energy Phys. 04 (2013) 130.

[77] R. D. Blandford and L. Hernquist, J. Phys. C 15, 6233 (1982).

[78] D. P. Menezes, M. B. Pinto, S. S. Avancini, A. P. Martínez, and C. Providência, Phys. Rev. C 79, 035807 (2009).

[79] P.-C. Chu, X. Wang, L.-W. Chen, and M. Huang, Phys. Rev. D 91, 023003 (2015).

[80] I. Ghisoiu, J. Möller, and Y. Schröder, J. High Energy Phys. 11 (2015) 121.

[81] P. Arnold and L. G. Yaffe, Phys. Rev. D 52, 7208 (1995).

[82] C. Bonati, M. D’Elia, M. Mariti, M. Mesiti, F. Negro, A. Rucci, and F. Sanfilippo, Phys. Rev. D 95, 074515 (2017).

[83] A. K. Rebhan, Phys. Rev. D 48, R3967 (1993).

[84] E. Shuryak, Phys. Lett. B 78, 150 (1978).

[85] S. Ghosh and V. Chandra, Phys. Rev. D 98, 076006 (2018).

[86] P. J. Silva, O. Oliveira, P. Bicudo, and N. Cardoso, Phys. Rev. D 89, 074503 (2014).

[87] R. A. Schneider, Phys. Rev. D 66, 036003 (2002).

[88] S. Mrowczynski, Phys. Part. Nuclei 30, 419 (1999).

[89] K. Yagi, T. Hatsuda, and Y. Miake, Quark-Gluon Plasma: From Big Bang to Little Bang (Cambridge University Press, Cambridge, 2005).

[90] M. Y. Jamal, S. Mitra, and V. Chandra, Phys. Rev. D 95, 094022 (2017).

[91] G. S. Bali, F. Bruckmann, G. Endrődi, and A. Schäfer, Phys. Rev. Lett. 112, 042301 (2014).

[92] J. Alexandre, Phys. Rev. D 63, 073010 (2001).

[93] B. Karmakar, A. Bandyopadhyay, N. Haque, and M. G. Mustafa, Eur. Phys. J. C 79, 658 (2019).

[94] S. Rath and B. K. Patra, J. High Energy Phys. 12 (2017) 98.

[95] A. Bandyopadhyay, B. Karmakar, N. Haque, and M. G. Mustafa, Phys. Rev. D 100, 034031 (2019). 